\title{
A 3D view of early mammals
}

\section{The unexpected discovery of a nearly complete skull from the Early Cretaceous epoch that has been preserved in three dimensions provides profound insights into the evolution and biogeography of early mammals. SEE LETTER P.108}

\section{SIMONE HOFFMANN \& DAVID W. KRAUSE}

$\mathrm{T}$ The fossils that advance our understanding of evolutionary history often come from parts of the world that have not been well studied by palaeontologists. Occasionally, however, game-changing fossils are discovered in heavily surveyed regions by targeting poorly sampled rock layers in those areas. On page 108, Huttenlocker et al. ${ }^{1}$ present one such discovery from the Cedar Mountain Formation in North America - an Early Cretaceous rock formation in Utah dated to between 139 million and 124 million years ago. The authors describe a complete, 3D fossil skull from a previously unknown genus and species, which they name Cifelliodon wahkarmoosuch.

Palaeontological fieldwork in western North America has led to the discovery of a greater number of Cretaceous mammal species and their more primitive relatives (collectively called mammaliaforms) here than in any other region in the world - more than 150 species have been found ${ }^{2}$. They are represented by tens of thousands of specimens, the vast majority of which are isolated teeth from the Late Cretaceous epoch (100 million to 66 million years ago). A few of the specimens are lower jaws, and many fewer are skulls or skeletons. The extreme rarity of 3D skulls makes Huttenlocker and colleagues' discovery momentous.

Aside from its 3D preservation (Fig. 1), the skull is remarkable in other ways. It is about 7 centimetres long, indicating that Cifelliodon was about the size of a medium hare. This would have been unusual in an Early Cretaceous world dominated by shrew- and mousesized mammals. Among its North American contemporaries, only one known species is larger - the carnivorous Gobiconodon ostrom $i^{2}$. In addition, the downturned face and relatively shallow snout make the skull unusual among early mammaliaforms.

Huttenlocker et al. used an imaging technique called micro-computed tomography $(\mu \mathrm{CT})$ to reveal a wealth of anatomical detail about the skull. For example, they found that Cifelliodon had a small brain with large olfactory bulbs. This combination is commonly seen in early mammaliaforms ${ }^{3}$, and is indicative of the keen sense of smell that is

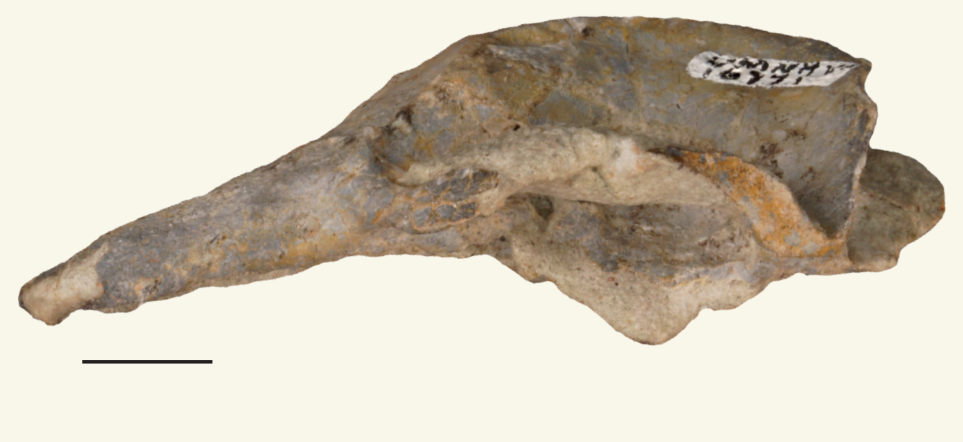

Figure 1 | A fossil from the dawn of mammals. Huttenlocker et al. ${ }^{1}$ report the discovery of a nearly complete skull from the Cedar Mountain Formation in North America, dated to between 139 million and 124 million years ago. They named the species Cifelliodon wahkarmoosuch, and suggest that it belongs to a group of animals called haramiyidans. The skull is shown in side view with the snout pointing left. Scale bar, 10 millimetres. (Image adapted from Fig. 1 of ref. 1.)

one of the hallmarks of mammals today.

Using the same technique, the authors also demonstrated that the bones of the occipital region at the back of the skull (in particular, the tabular bones) remained unfused in Cifelliodon. Unfused tabulars are a rare trait in mammaliaforms - the tabulars are generally fused with the occipital bones in adult mammals, but remain separate in other vertebrates ${ }^{4}$. But, as the authors point out, the supposition that these bones are fused in early mammaliaforms could be biased by the rarity of well-preserved skulls, or by the techniques used to analyse them. The few mammaliaforms that have been found to have unfused tabular bones, as in Cifelliodon, are known from 3D skulls or have been scanned using $\mu \mathrm{CT}^{1,5}$, which enables the detection of cranial structures that might be invisible on the skull's surface.

The completeness of the Cifelliodon skull allowed Huttenlocker et al. to assess the early branches of the mammaliaform family tree more robustly than has previously been possible. This phylogenetic analysis places Cifelliodon in the extinct Haramiyida - a group that has elicited controversy because its position in the evolutionary tree has implications for the timing of the origin of mammals.

Until five years ago, the fossil record of haramiyidans consisted mainly of isolated teeth from a few European sites from the Late Triassic (237 million to 201 million years ago) and
Early Jurassic (201 million to 174 million years ago), and a lower jaw and a few postcranial bones from a Late Triassic site in Greenland ${ }^{2}$. In 2013, two almost-complete haramiyidan skeletons from the Middle Jurassic (174 million to 164 million years ago) were discovered in China, sparking controversy about mammalian relationships ${ }^{6,7}$. The two research groups involved came to very different conclusions: one placed Haramiyida in Mammalia ${ }^{6}$, the other outside it ${ }^{7}$. This difference is not trivial: it results in vastly different temporal estimates for the origin of mammals.

Placing Haramiyida in Mammalia pushes the origin of the latter group back to the Late Triassic (215 million years ago). Such a date would imply that several mammalian clades, including the lineage that led to placental and marsupial mammals, have earlier origins than was thought. This earlier date also implies that there were long intervals of time in which these early lineages were present but for which fossils have not yet been found.

By contrast, placing Haramiyida outside Mammalia suggests an origin in the Early Jurassic (about 185 million years ago). This implies a relatively explosive diversification of early mammals.

Several further haramiyidans from the Jurassic period have since been discovered in China, but the controversy has only intensified $^{5,8-10}$. Particularly problematic is the fact 


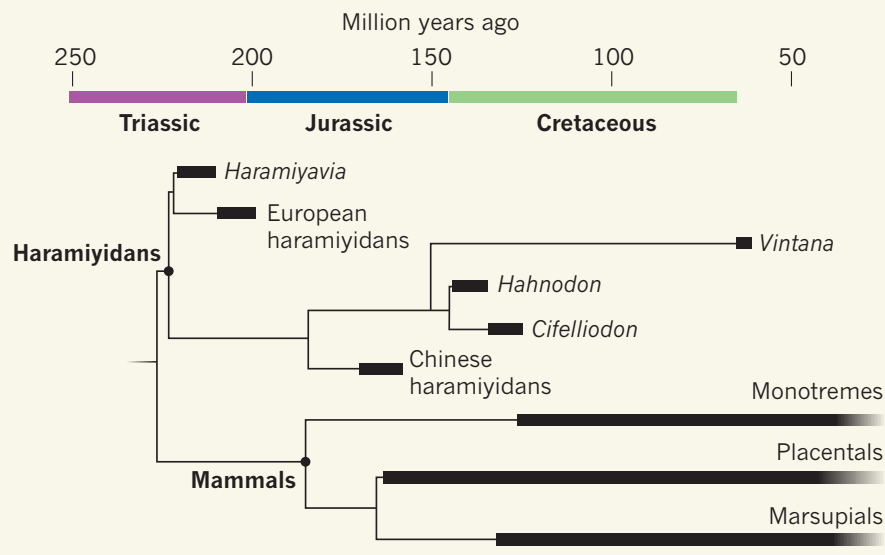

b

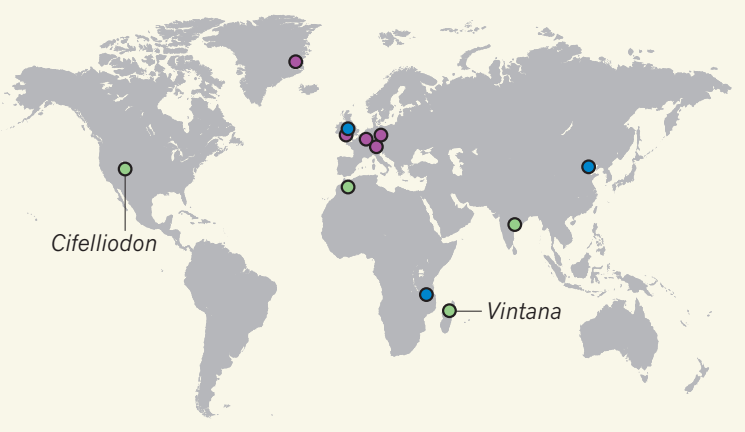

Figure 2 Re-evaluating the evolution and biogeography of haramiyidans. a, Huttenlocker $\mathrm{et} \mathrm{al.}{ }^{1}$ analysed relationships between the early branches of the family tree for mammals and their more primitive relatives. The resulting evolutionary tree indicates that haramiyidans are not mammals, contrary to some previous evidence ${ }^{5,6,8,9}$. The analysis also places the Cretaceous genus Vintana in Haramiyida for the first time. b, Cretaceous haramiyidans (indicated by green circles) have previously been found in northern Africa and possibly
India. The authors' analysis expands the Cretaceous range of haramiyidans to Madagascar (Vintana) and North America (Cifelliodon). Combined with the fact that other fossils of haramiyidans from the Triassic (purple) have been found in Europe and Greenland, and that haramiyidans from the Jurassic (blue) have been found in Europe, China and Tanzania, this work implies a much broader temporal and geographical distribution of haramiyidans than had previously been hypothesized. that, although the Chinese haramiyidans are represented by complete skeletons, the specimens are essentially 2D. Most of the skulls are little more than flattened outlines, which limits their usefulness for informing mammalian relationships.

Cifelliodon is one of the first skulls preserved in three dimensions from the haramiyidan lineage. As such, it is a crucial piece of the evolutionary puzzle. Huttenlocker and colleagues' phylogeny puts Haramiyida (and so Cifelliodon) outside Mammalia (Fig. 2a). Thus, their work favours a model in which early mammals diversified rapidly during the Jurassic.

Finally, Huttenlocker et al. provide evidence that Cifelliodon is closely related to Cretaceous species from northern Africa (Hahnodon taqueti) and Madagascar (Vintana sertichi), the latter of which had not previously been assigned to Haramiyida. This implies a much broader temporal and geographical distribution for Haramiyida than has been assumed (Fig. 2b), indicating the need to reassess the biogeographical history of the group. The authors conclude that haramiyidans had a global distribution during the JurassicCretaceous transition, and that land bridges aiding vertebrate dispersal existed long after the fragmentation of the supercontinent Pangaea - much later than previously recognized. An alternative hypothesis that is perhaps more consistent with current palaeogeographical models ${ }^{11}$ is that haramiyidans, like many vertebrate groups, had a Pangaean distribution in the Jurassic period and evolved in isolation thereafter, as landmasses separated during the Cretaceous period. The best way to test these competing hypotheses is with the discovery of more well-preserved fossils, like this exquisite skull of Cifelliodon.
Simone Hoffmann is in the Department of Anatomy, New York Institute of Technology, College of Osteopathic Medicine, Old Westbury, New York 11568, USA. David W. Krause is in the Department of Earth Sciences, Denver Museum of Nature \& Science, Denver, Colorado 80205, USA. e-mails:shoffm04@nyit.edu; david.krause@dmns.org

1. Huttenlocker, A. K., Grossnickle, D. M., Kirkland, J. I., Schultz, J. A. \& Luo, Z.-X. Nature 558, 108-112 (2018).

2. Kielan-Jaworowska, Z., Cifelli, R. L. \& Luo, Z.-X. Mammals from the Age of Dinosaurs: Origins, Evolution, and Structure (Columbia Univ. Press, 2004).
3. Rowe, T. B., Macrini, T. E. \& Luo, Z.-X. Science 332 955-957 (2011).

4. Koyabu, D., Maier, W. \& Sánchez-Villagra, M. R. Proc. Natl Acad. Sci. USA 109, 14075-14080 (2012).

5. Krause, D. W. et al. Nature 515, 512-517 (2014)

6. Zheng, X., Bi, S., Wang, X. \& Meng, J. Nature 500, 199-202 (2013).

7. Zhou, C.-F., Wu, S., Martin, T. \& Luo, Z.-X. Nature 500, 163-167 (2013).

8. Bi, S., Wang, Y., Guan, J., Sheng, X. \& Meng, J. Nature 514, 579-584 (2014).

9. Han, G. et al. Nature 551, 451-456 (2017).

10.Luo, Z.-X. et al. Nature 548, 326-329 (2017).

11.Torsvik, T. H. \& Cocks, R. M. Earth History and

Palaeogeography (Cambridge Univ. Press, 2017).

\title{
ASTRONOMY
}

\section{A fresh approach to stellar benchmarking}

\begin{abstract}
An avalanche of data is about to revolutionize astronomy, but the options for validating those data have been limited. High-precision measurements from the Hubble Space Telescope enable a much-needed alternative option.
\end{abstract}

\section{RACHAEL BEATON}

1 ry this experiment: extend your thumb at arm's length and close one eye at a time. Your thumb will seem to 'jump' between two positions as you switch the eye that is closed. That jump is known as parallax. If you measure the jump as well as the distance between your eyes, you can use trigonometry to calculate the distance to your thumb. Astronomers use parallax, on a much greater scale, to measure distances to astronomical objects. Writing in The Astrophysical Journal Letters, Brown et al. ${ }^{1}$ report that they have achieved this for the nearby star cluster NGC 6397, using the Hubble Space Telescope. Their method will provide a crucial means of validating the wealth of parallax data released this year from the European Space Agency's Gaia mission ${ }^{2}$.

It is a challenge to find a topic in astronomy that does not rely on the astronomical distance 